\title{
Diagnostics For Recirculating And Energy Recovered Linacs ${ }^{1}$
}

\author{
Geoffrey A. Krafft and Jean-Claude Denard ${ }^{2}$ \\ Thomas Jefferson National Accelerator Facility \\ 12000 Jefferson Ave., Newport News, VA 23606
}

\begin{abstract}
In this paper, the electron beam diagnostics developed for recirculating electron accelerators will be reviewed. The main novelties in dealing with such accelerators are: to have sufficient information and control possibilities for the longitudinal phase space, to have means to accurately set the recirculation path length, and to have a means to distinguish the beam passes on measurements of position in the linac proper. The solutions to these problems obtained at Jefferson Laboratory and elsewhere will be discussed. In addition, more standard instrumentation (profiling and emittance measurements) will be reviewed in the context of recirculating linacs. Finally, and looking forward, electron beam diagnostics for applications to high current energy recovered linacs will be discussed.
\end{abstract}

\section{INTRODUCTION}

Small recirculating electron accelerators, operating at beam energies below $1 \mathrm{GeV}$, have existed for many years starting with the development of the microtron. In the past decade, several GeV scale recirculating accelerators have been built, including the Bates recirculating linac at MIT, the Mainz cascaded racetrack microtrons, and the 5pass superconducting recirculating linacs at Jefferson Lab. These accelerators have now operated long enough that many standard beam diagnostic techniques have been used and developed, and it is worthwhile to review the progress that has been made on new, and not so new, beam diagnostic elements.

A schematic diagram of the Continuous Electron Beam Accelerator Facility (CEBAF) is shown in Figure 1, and a summary of the main accelerator parameters is given in Table 1. CW beam, originating in an injector, is recirculated up to five times through each superconducting radiofrequency (SRF) linac. The beam may be directed into up to three experimental halls simultaneously, the beam current in each hall being at the third subharmonic of the fundamental RF frequency of $1497 \mathrm{MHz}$. The lowest operating energy is about $0.8 \mathrm{GeV}$, the present full energy is nearly $6 \mathrm{GeV}$, and a costeffective upgrade to $12 \mathrm{GeV}$ is possible and planned. The combination of five-pass recirculation, a three-laser photocathode source, and subharmonic-rf-separator-based extraction enables simultaneous delivery of three beams at different but correlated energies.

\footnotetext{
${ }^{1}$ Work supported by United States Department of Energy under contract DE-AC05-84ER40150.

${ }^{2}$ Present Address: Synchrotron Soleil - Bat. 209H - Centre Universitaire Paris Sud - BP34 - F 91898 Orsay Cedex
} 


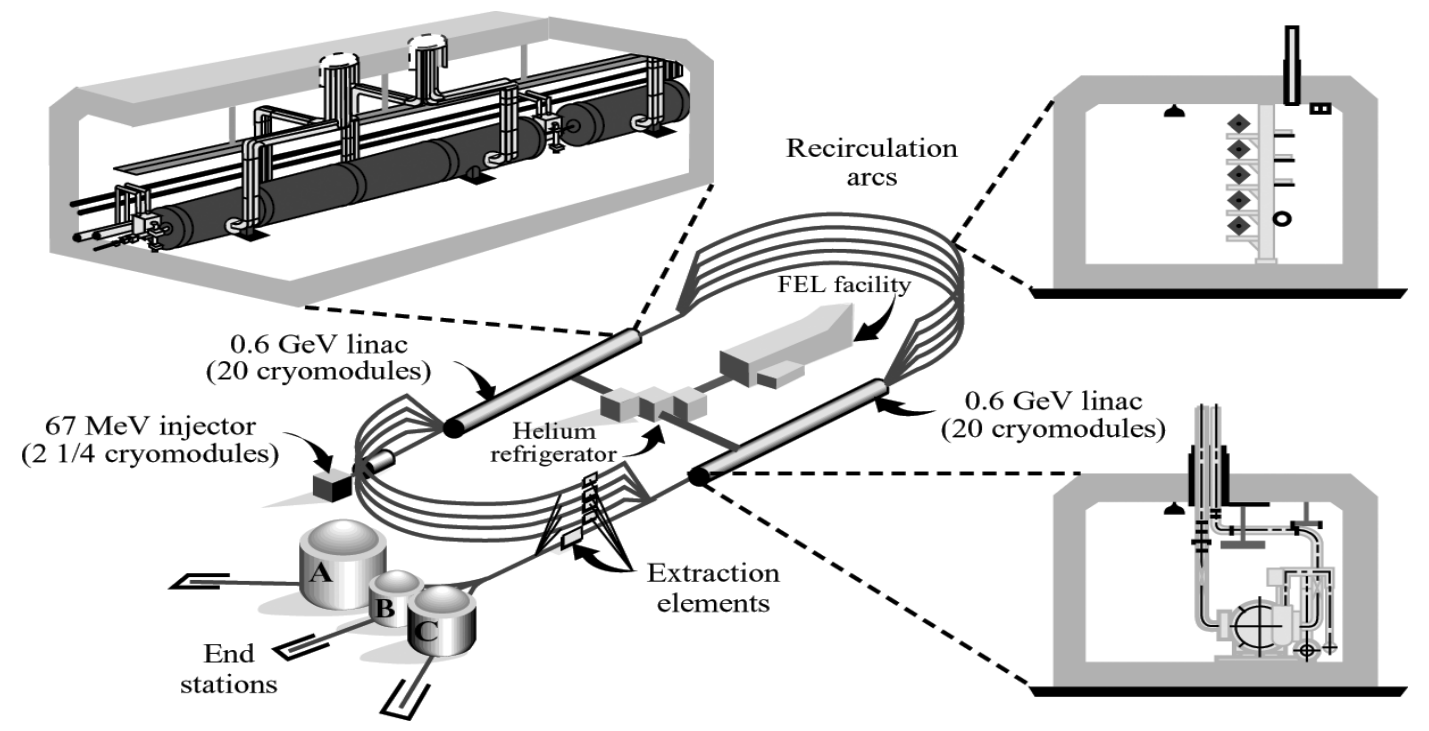

FIGURE 1. Jefferson Laboratory's CEBAF accelerator.

The most radical innovations in CEBAF are the choice of SRF technology and use of multipass beam recirculation. Neither had been previously applied on the scale of CEBAF. In fact, until LEP II came into operation, CEBAF was the world's largest implementation of SRF technology. Beam recirculation minimizes the cost of the SRF linacs to achieve a given energy, and has been executed with large enough bend radii to keep open the possibility of future energy upgrades.

TABLE 1. CEBAF Accelerator Parameters.

\begin{tabular}{lcc}
\hline Item & Value & Unit \\
\hline Beam Energy & $0.8-6$ & $\mathrm{GeV}$ \\
Beam Average Current & $<180$ & microAmps/Hall \\
Normalized $r m s$ Emittance & $<1$ & $\mathrm{~mm} \mathrm{mrad}$ \\
Repetition Rate & 499 & $\mathrm{MHz} / \mathrm{Hall}$ \\
Charge per Bunch & $<0.4$ & $\mathrm{PC}$ \\
Extracted $r m s$ Energy Spread & $<10^{-4}$ & \\
Transverse $r m s$ Beam Size & $<100$ & microns \\
Longitudinal $r m s$ Beam Size & $60(200)$ & microns(fsec) \\
Beam $r m s$ Angular Spread & $<1 / \gamma$ & \\
\hline
\end{tabular}

As shown in Table 1, recirculated linacs like CEBAF can be designed for short bunch operation naturally, because the beam orbit does not actually close on itself as in a ring. Many techniques for measuring, monitoring and adjusting bunch length have been developed as part of the project, allowing routine operation of this accelerator at bunch lengths of order $100 \mathrm{fsec}$. When it comes to actually operating a recirculated linac, some of its properties must be regulated in ways somewhat differently than in typical linacs or rings. Therefore, it is worth sharing some of the techniques found useful for stabilizing and maximizing beam performance in such accelerators. 
In addition to such high energy accelerators, increasing interest in high current recirculating accelerators, particularly those that are energy recovered, is developing. At the moment, the highest average current recirculated linac in existence is the Jefferson Lab Infrared Free Electron Laser (IRFEL). An energy recovered arrangement was chosen for this accelerator because it has long been realized that energy recovered operation may provide the only path to high average power (MW scale) in an FEL, and the idea needed to be explored quantitatively on a relatively small-scale recirculated linac. Two attendant benefits of choosing to energy recover this particular linac were: (1) the RF power required to continuously run the FEL is greatly reduced, and (2) the disposal problems associated with the spent electron beam after the FEL interaction is considerably alleviated. The device proved to be a useful test stand for developing beam diagnostics, as will be mentioned in the body of this work.

In this paper we will discuss longitudinal phase space measurement, manipulation, and control; recirculation path length adjustment; multipass beam position monitoring; and operational control ideas, especially data obtained from rapid, time synchronized toggling measurements. Next, options for beam profiling in such devices are given. The paper concludes with an introduction to the types of advanced diagnostics that may be required for future high current recirculated linacs.

\section{LONGITUDINAL DYNAMICS AND PHASE SPACE MEASUREMENTS}

Unlike the Bates recirculator, where the beam energy spread requirement does not dictate the need for short bunches, or the Mainz racetrack microtrons, where the primary determinants of the extracted longitudinal phase space are the initial matching and the longitudinal stability of the phase oscillations in the accelerator, the CEBAF accelerator from the beginning was designed to have an extremely small relative energy spread [1]. The approach taken in the design was to: (1) design a continuous beam electron injector that produced very short bunch lengths, (2) design recirculation optics that were isochronous $\left(R_{56}=0\right)$ and achromatic, and (3) choose to operate the linacs on the crest of the accelerating wave. There is no longitudinal focusing in the accelerator proper, and small single bunch energy spread arises from the small electron pulse length interacting with the curvature of the crested RF wave, assuming each of the beam passes is made at the same RF phase. In this section we discuss how to make and observe the short bunches, in the next we discuss how to make the different beam passes have simultaneous time-of-arrival.

In a recent review, the requirements on bunch length for producing a small energy spread are given [2]. In order to have less than $2.5 \times 10^{-5} \mathrm{rms}$ energy spread from the RF curvature, and to have some control margin to allow fast fluctuations in the RF controls and the energy spread that they generate, a bunch length of $1.8 \mathrm{RF}$ degrees ( 3 psec $r m s$ ) was specified for CEBAF. In the course of detailed longitudinal matching designs of the injector it was found that bunch lengths as short as 0.1 degree (200 fsec $r m s$ ) were possible, and of course desirable as they reduce the overall single bunch energy spread. 
Three somewhat different types of measurements were done in order to verify such short bunches are possible and have been obtained at CEBAF. The first, both chronologically and with greatest utility operationally, was the phase transfer monitor [3]. Its principle and operational use are discussed at length and quite thoroughly elsewhere [4]. In brief, a longitudinal beam pick-up is used to obtain a beam derived $\mathrm{RF}$ signal that is mixed against the RF master oscillator to detect time-of-arrival. By modulating the beam chopping phase and simultaneously detecting and recording time-of-arrival, the phase transfer technique is used to produce a graph of output bunch phase against input bunch phase, including the non-linearities in the transfer response. By properly correcting nonlinear behaviors in the transfer function plots, one is in reality correcting and adjusting non-linear distortions in the longitudinal phase space. When properly done, all the different longitudinal slices of the longitudinal phase space at the chopping point are adjusted to have the same time-ofarrival at the location of the pick-up. The pick-up cavity is strategically placed just prior to the first acceleration to $45 \mathrm{MeV}$, after which little further longitudinal motion arises due to relativistic effects. The resolution of the technique is below $200 \mathrm{fsec}$, which corresponds to 60 microns in bunch length.

Chronologically the next method developed was a monitor based on coherent synchrotron radiation (CSR) [5-7]. From a continuous operation and monitoring point of view, non-invasive beam diagnostics are preferred, and those based on measuring synchrotron light are preferred to those based on transition radiation for this reason. Because the bunch length is of order 100 microns fully compressed, and because the linac beam must be bent through an injection magnetic chicane anyway, narrowband coherent synchrotron radiation detection was used in order to provide a continuous bunch length (change) monitor. CSR was detected through a bandpass filter with center wavelength of 500 microns and transmission width of $20 \%$. Such a monitor, based on sensitive Schottky diode detection, was able to detect signals at down to 1 microA beam current easily [8]. Some care must be taken when applying techniques based on spectrum detection of bunch shapes, because it is actually highly unlikely that the bunch shapes in linacs, recirculating or not, are Guassian, or that calculations of the bunch shape based on Guassian distributions will lead to meaningful results $[5,9]$. So even at the earliest stages of the CEBAF work on coherent detection techniques, it was understood that some other method would be needed to obtain the bunch shape accurately.

The third method used to analyze the bunch length and distribution shape was the so-called zero phasing method. As indicated schematically in Figure 2, in this time domain method, one (or several) RF accelerating cavities are phased close to the zerocrossings of the accelerating fields, and the beam longitudinal distribution is analyzed by transverse measurement by a beam profile monitor located at a location of non-zero dispersion [10]. Data at both crossings and with the RF in the zero-phased cavities off is gathered, and differences in the observed distributions between positive going and negative going crossings can be used to infer information about the slope of the longitudinal phase space distribution. At CEBAF, zero-phasing measurements are considered the standard baseline technique, from which the CSR monitor could be calibrated for an actual bunch length measurement. Such cross calibrations apply to the extent that distribution shape changes are small during the period of monitoring, a 
circumstance that can only be verified a posteriori, by zero-phasing measurements before and after the period of beam monitoring.

At Jefferson Lab, the smallest bunch length, determined by a Guassian fit to zerophasing longitudinal distribution data, was $85 \mathrm{fsec} r m s$. It was verified that CSR emission was highest when the bunch length was shortest in this machine setup [11]. Many years after this work, it was verified that the standard operations mode for the CEBAF accelerator has $r m s$ bunch length from 150-200 fsec, over the entire current operating range in the accelerator, well below the requirements [12]. The main disadvantage of the zero-phasing method is that it is highly invasive to normal beam operations because special RF setups have to be introduced to make the measurements. On the other hand, the information that is derived from the method is quite detailed as to the actual longitudinal distribution. Recently the method has been used to determine time dependent beam parameters (slice parameters) from the beam emerging from an RF gun at Brookhaven National Laboratory. The time resolution reported was $8 \mathrm{fsec}$ in a bunch whose duration is several psec [13].

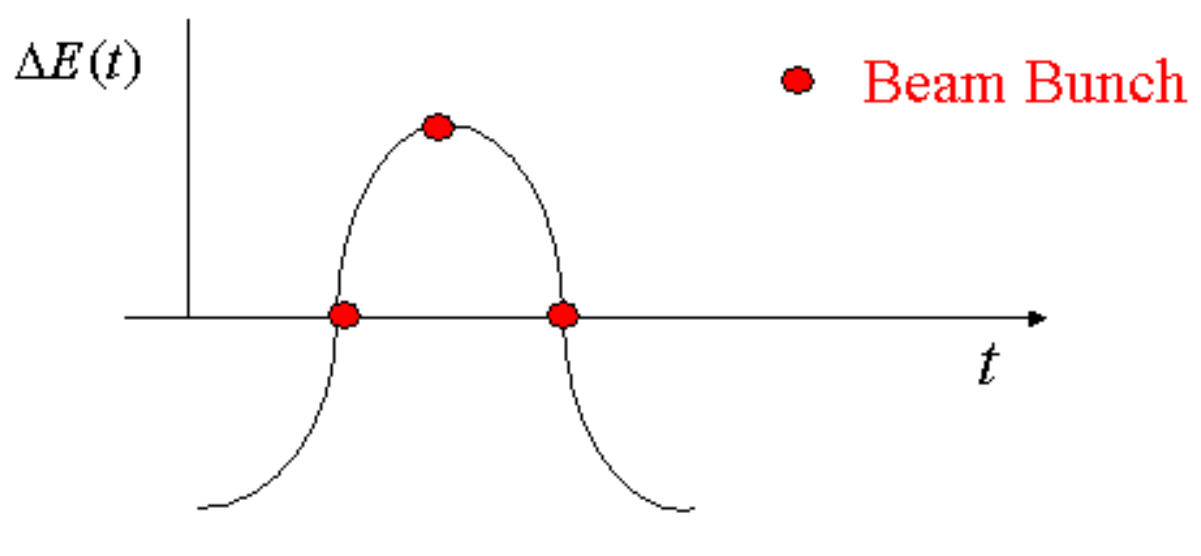

FIGURE 2. RF settings for zero phasing measurements of bunch length.

\section{RECIRCULATION BEAM PATH}

The next quantity that must be properly set is the recirculation beam path. The requirements are again given in Reference [2]. In order to have a small contribution to the energy spread of the beam, each pass of the beam must have the time of arrival within a few degrees of the energy crest phase for all the passes simultaneously. One way to achieve this condition is to: (1) measure and crest the bunches passing through each of the accelerators in CEBAF on the first pass, followed by (2) making sure that each bunch of each subsequent pass arrives at the same time as the first pass beam within the requirement. This approach is taken in the chronologically first method of path length control discussed next. Presently, a newer, non-invasive and continuous method of path length control is being investigated and implemented and will be described in the following subsection. Those readers used to storage ring practice of 
adjusting the accelerator frequency to adjust for path length shifts should recall that such an idea is probably straightforward to implement on a two pass recirculated linac, but is not of obvious utility for more passes as the differing path lengths of the passes are likely to be unequal, and not adjusted properly by a single frequency change. It also might be operationally challenging to be adjusting the operating frequency of many superconducting cavities on a continuous or semi-continuous basis.

\section{Direct Measurements By Time Of Arrival}

The first method for path length control involved periodic cresting of the bunches on the accelerating fields of the linacs in the first passes, followed by precision relative time-of-arrival monitoring of the higher pass beam bunches. Cresting, which presently happens no more frequently than once a day during quiet operations, now proceeds automatically [14]. No additional beam instrumentation is need beyond a $\mathrm{BPM}$ or viewer located in a dispersed location for observation of the crest condition.

Special instrumentation was developed for the higher pass relative time-of-arrival monitoring that is based on the phase transfer measurement electronics. The outputs from longitudinal pickup cavities installed inside the recirculation loop are amplified and fed into one side of several fundamental frequency mixers, the other sides being fed by a fundamental frequency signal derived from the master oscillator. The first signal can be phase shifted (electronically) so that on average, the mixer acts as a phase detector.

A special beam condition is established to perform the measurement. The once around time for beam in CEBAF is 4.2 microseconds. If a 4 microsecond beam pulse goes through the 5 passes of the accelerator, the signal out of the longitudinal pickup will come in successive 4 microsecond chunks spaced by 4.2 microseconds, one for each pass of the machine [15]. Ideally, when all the arrival times are identical, the output from the phase detector is constant and zero if the phase shifter is properly set. If there are level changes in the output of the phase detector, one can easily infer the beam phase shift on the pass of interest. In theory, it merely remains to digitize the signal out of the phase detector at the correct time corresponding to each pass. In current practice, the device is a little more accurate because the full waveform out of the phase shifter is digitized at $10 \mathrm{MHz}$, and the phase shift levels are determined by software analysis of the complete record. Accuracy is increased because many data points are averaged to determine the phase shift of each pass [16].

The performance of this device is very good. Even at the standard measurement mode operating current of $3 \mu \mathrm{A}$, the precision is 0.1 degrees or $200 \mathrm{fsec}$ in the individual readings. Averaging within a single pass waveform increases the precision by about 5 . These measurements correspond to changes in the machine circumference of about 10 microns.

\section{Beam Based Phasing By Phase Modulation}

The main weakness of the direct method of phase error determination is that it requires the machine to be placed in a pulsed beam mode and is therefore invasive to normal beam delivery. It would be far better to have a non-invasive method of phase 
monitoring that can operate with the standard $\mathrm{CW}$ beam delivered to users. Because of the development of feedback systems that operate at high enough sample rate [17], it is possible to consider placing various modulations on the beam, that will be removed before delivery of the beam to users, but which also have beam diagnostic purposes. In fact, the best way to check that one is on crest of a linac is to phasemodulate the linac and make sure that the energy change is zero for each pass of the beam through each linac. As shown schematically in Figure 3, this principle is used for the continuous phase monitoring capability that has been recently developed at CEBAF [18]. If the bunch is crested, as shown in the Figure, there is no energy change at the modulation frequency, and a small signal at twice the modulation frequency due to the curvature of the RF wave on crest (the modulation amplitude is expanded for clarity in Figure 3). On the other hand if there is in fact a phase offset between the beam and the linac RF, there is an energy modulation produced by the phase modulation at the modulation frequency, that is to first order linear in the phase displacement.

In order to obtain information that discriminates errors that arise in the separate CEBAF linacs, the phases of the two linacs are modulated at two different frequencies: $383 \mathrm{~Hz}$ and $397 \mathrm{~Hz}$. In practice, the amplitude of modulation is small enough (0.05 degrees at $1497 \mathrm{MHz})$ that the feedback system can adjust away the energy centroid shift, and small enough that there are no other deleterious effects generated by the modulation. Utilizing lockin techniques to generate the phase modulations, BPMs at dispersed locations in each pass beamline at the end of the second CEBAF linac are observed for beam motion at the lockin frequencies. After calibration, observed lockin outputs correspond to the summed phase shifts (from all previous passes) from each linac. As there should be no phase shift anywhere, a simple matrix calculation gives the individual phase shifts to be adjusted on each pass [19].

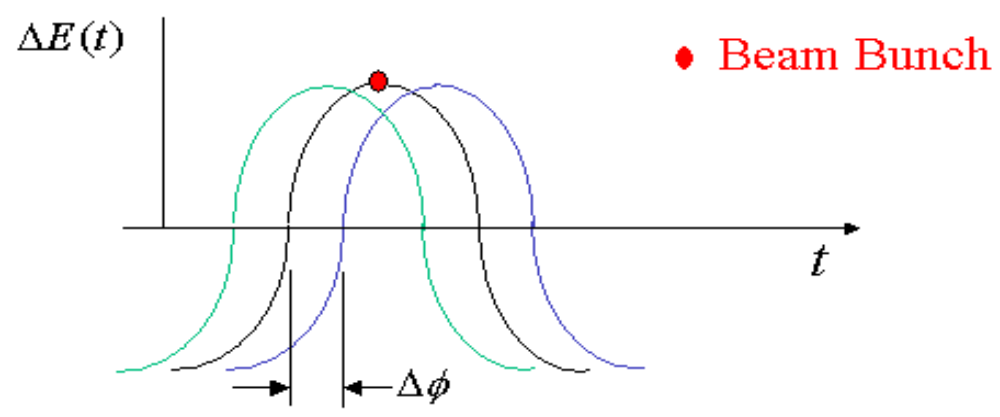

Bunch "Crested" when $d \Delta E / d t=0$

FIGURE 3. Beam based phasing by linac phase modulation. The modulation amplitude $\Delta \phi$ is 0.05 degrees at $1497 \mathrm{MHz}$. The beam phase offset is proportional to the same-frequency energy modulation. 


\section{MULTIPASS POSITION MEASUREMENTS}

As the beam propagates along the linac, each beam pass present at a beam position monitor excites the device. Ideally, the beam position monitor should be able to give the user information on the beam position on individual passes. How is the individual pass information to be obtained? Four basic methods have been suggested to solve this problem: (1) measure properly timed beam pulses (or gaps!), (2) distinguish individual passes by time-of-arrival of the beam pulses originating on the monitor electrodes, (3) utilize pseudorandom sequences with orthogonal time delay properties combined with beam modulation to separate out signals from the different beam passes, and (4) measure positions with monitors of two different frequencies.

The first method involves providing a beam pulse and reading the beam position monitor electronics at a time when only one beam pass is in the monitor. Thus, the measurement is much like the direct time-of-arrival measurement discussed above. As long as the detection electronics have response times short compared to the oncearound time, which is 4.2 microseconds for CEBAF, measurement and interpretation of the results is straightforward. A variant, that is better for application to a high duty factor accelerator, but is slightly more complicated to analyze and calculate and has reduced dynamic range, is to blank off the beam for times short compared to the once around time, and synchronize the digitization of the monitor electronics with the arrival of the "gap" on the different passes [20]. It should be noted that in order for this method to succeed, the BPM trigger or reading system must have a means to delay the read time to account for the fact that individual monitors should be read at different times at different longitudinal locations in the accelerator.

The second method, at this stage mainly theoretical, applies only in cases where the charge-per-bunch is high enough that measurable beam pulses are produced on the BPM electrodes, and in situations, as in the IRFEL, where there is a substantial time difference between the accelerating beam pass and decelerating beam pass because only a small fraction of the accelerating buckets are filled. Because the accelerating and decelerating beam pulses are separated in time, a sufficiently precise timing method is able to discriminate the information from the separate beam passes. Such a method is, from a timing point of view, much more sensitive to time jitter between digitization trigger and beam arrival than in the first method; now the relevant time scale to distinguish the bunches is the time of bunch separation, not the once around time. Measurements on the Jefferson Lab IRFEL have shown that accelerating and decelerating beam pulses are indeed distinctly observable on an oscilloscope, but the next-level design of a suitable detection and digitization system has not been completed at this time. For accelerators designed with more than two passes, it will be essential to have the various accelerating and decelerating passes in different RF buckets in order to apply this method.

The third method is probably the best method to apply to diagnosing $\mathrm{CW}$ beam, but is the most difficult conceptually and the most difficult to implement. It has long been known that various bit sequences may be produced that are pseudorandom (equal numbers of zeros and ones) with various beneficial properties in addition. In particular, it is possible to produce sequences that when digitally mixed with themselves yield non-zero output, but when mixed with time-delayed versions of 
themselves yield zero output. As the once-around time from the accelerator produces a fixed time delay between the beams on different passes for a machine like CEBAF, an obvious application of such sequences is to modulate the beam current at a small level in a pseudorandom way where the "states" change once in a once-around time, and detect electrode readings once in a once-around time. The data stream is analyzed by mixing (both analogue and digital mixing have been proposed) the data stream with the pseudorandom code synchronized with the first pass beam to get the first path beam position information, by mixing the data stream with the pseudorandom code shifted by the once around time to get the second pass beam position information, by mixing the data stream with the pseudorandom code shifted by twice the once around time to obtain third pass beam information, and so on. Of course the code must become longer the larger the number of passes and the time response of the monitor will become correspondingly longer as the code length increases.

A final possibility, that is particularly clean when applied to an energy recovered linac (ERL) with one accelerating pass and one decelerating pass and every bucket filled, is to have two sets of BPMs at different frequencies (or a single BPM that can detect both fundamental and second harmonic): the fundamental and twice the fundamental. It is clear that if the position of the two beam passes is identical, then the fundamental is not excited at all and twice the fundamental is excited with an amplitude twice as large. On the other hand, if the two beams were displaced oppositely, the fundamental would be excited and the second harmonic would be zero. So by taking a slightly more complicated difference over sum formula, both positions of both beams may be determined. Note that in general the sensitivity of the BPMs at the different frequencies might be quite different and must be adjusted for.

Up to now, only method 1 above has been used at CEBAF. It clearly is of no help for continuous monitoring of the $\mathrm{CW}$ beam delivered during normal beam operations.

\section{FAST DIFFERENCE MEASUREMENTS}

From a beam diagnostics point of view, one of the advantages of a recirculated linac is that the beam orbit does not close on itself. Therefore, the process of verifying the beam optics of the focusing lattice can be somewhat simpler than in rings. Most of the optics are measured utilizing some sort of differencing scheme. For example, difference orbit measurements throughout the accelerator are performed by activating sets of steerers in the accelerator and observing the beam orbit changes on beam position monitors (BPMs). Likewise, the dispersion characteristics of the bend regions and arc may be evaluated by taking differences in the orbits between two separate energy states. Another example in the longitudinal direction, the isochronicity of the bending arcs may be evaluated by measuring the time-of-arrival phase at two different beam energies. In the earliest days of the CEBAF accelerator, these types of measurements were either done by hand changes in the perturbing elements, or by gathering data using simple computer scripts to make the changes and record the data for off-line analysis [21]. The idea, similar to the old batch processing methods on computers, was that only after analysis would one make adjustments to

the accelerator. Such a scheme, because of the time it takes and because of the 
difficulty in maintaining beam conditions between measurements, becomes totally impractical for any adjustments except the simplest, and can severely limit ones ability to do more sophisticated types of correction algorithms.

Following the computer analogy, more modern interactive computer programs based on real time systems were developed to allow users to do work where one could easily change parameters and observed changes in the results calculated by the computer. Such an approach is effective to the extent that all calculations are completed on a time-scale short compared to a human response time because one reduces the dead time of the human user. The fast optics difference measurements were developed to be rapid enough that an operator (user) can perform adjustments and observe results in real-time so that he/she can quickly converge to an accelerator condition that is known to give reliable beam operations.

The earliest attempts at fast differencing occurred during the commissioning of the first CEBAF arc. In order to determine the $R_{56}$ of the first arc, which should nominally be zero, a $70 \mathrm{~Hz}$ square-wave modulation was placed on the amplitude of a linac cavity and the time-of-arrival was observed with an RF phase detector [21]. As the pulsed tuneup beam was line synched, the output from the phase detector consisted of two levels corresponding to the phases of the two energy beams. Setting $R_{56}$ to zero could be done in real time by adjusting magnets in the arc beam transport system that were at positions of non-zero dispersion so that the phase difference was zero. In addition, it was checked that two spots appeared on viewers at positions with non-zero dispersion corresponding to the two energy states when the modulation was present. This means an easy way to set the dispersion to zero, a step needed as part of machine setup, is to modulate and adjust the quadrupoles that control dispersion so that two spots become one. An operator can perform this chore rapidly, because of the realtime nature of the response of the accelerator.

A more quantitative system was developed based on $30 \mathrm{~Hz}$ toggling of the $60 \mathrm{~Hz}$ tune-up beam [22]. For difference orbits, the beam is toggled between two states with two sets (one for each transverse direction) of air-core magnets, and for dispersion the beam is toggled in two energy states. The data acquisition is synchronized to the toggling, so the sign of the dispersion or matrix element is unambiguously determined at the location of every beam position monitor in the machine. The dispersion adjustment is easy conceptually: the operator must zero out dispersion outside any bend region of the accelerator. Setting up a confined difference orbit (and by implication a confined beam orbit) is done by calculating the difference between the Courant-Snyder invariant measured by the toggling system, and the design invariant, and doing adjustments so that the growth in amplitude is minimized. Through the use of this system, all nine passes of the accelerator optics can be adjusted so that there is no growth in Courant-Snyder invariant in a period of about an hour. After performing this procedure, it is almost always the case that the measured machine aperture (as measured by increasing the amplitude of the toggling steerers) is quite large compared to the beam size, and the beam optics is properly contained.

To complete this section, it should be mentioned that in addition to the benefit to the operator, having fast differencing capability allows optics experts to make measurements far more complete, thorough, and sophisticated with the toggling 
system than without it. This type of work, which tends to be "batch mode" due to limited machine development time, proceeds more rapidly if greater quantities of relevant data may be gathered within a given time period.

\section{BEAM PROFILING TECHNIQUES}

In this section references are given to some of the beam profiling techniques used on the CEBAF accelerator. Almost all of the beam viewers in CEBAF are made from phosphorescent materials, and all such beam viewers in the linac regions where multiple beam passes are on a common beam line have a transverse hole near the center of the viewer. Having such a hole is important to allow lower pass beams through the viewer in an unobstructed and unperturbed way, while retaining the ability to observe the higher pass beams. The lower pass beams are merely cleanly steered through the viewer holes. The advantages of phosphorescent viewers are that a common material and viewer design work throughout the machine energy range in its most useful application: observing whether beam halos or tails have formed on the beam, and which are usually corrected through the machine optics checks mentioned above, or by measurement and correction of RF phases in the injector. The disadvantages of the viewers are two-fold. The main disadvantage is that such a viewer cannot remain inside the $\mathrm{CW}$ electron beam without the viewer being destroyed. Consequently, only a pulsed low duty factor "tune-up" beam can be analyzed with these devices. Even if we would like to analyze the tune-up beam in detail, it is well known that phosphorescent devices are easily saturated, leading to ballooning of the beam distribution on measurement.

Both of these problems are "solved" by implementing optical transition radiation (OTR) viewers. Because the OTR radiator emits radiation prompt with the transition and the light propagates only in vacuum, and because the optical power emitted is much lower than for phosphorescent devices to begin with, the saturation characteristics and the linearity of the emission with beam current of the transition radiators are much superior, making the development of a profiling instrument somewhat easier and more accurate. It has also been possible to develop foils thin enough, the foils at CEBAF are $1 / 4$ micron in width, so that the electron beam is practically unperturbed by its interaction with the radiator, and so that the radiator easily survives the maximum $\mathrm{CW}$ beam current in the accelerator [23].

OTR viewers were first installed at CEBAF to perform an experiment to verify that the resolution limits of such viewers did not go as $1 / \gamma$, as had been postulated. In fact, a $4 \mathrm{GeV}$ beam was analyzed with an OTR viewer with a resolution of 60 microns, where the camera was sensitive from 300-600 nm [23]. After this experiment was successfully concluded, most subsequent viewer installation, which was usually motivated by the desire to perform a detailed profile measurement, has been of OTR devices. That the OTR viewers could monitor high current $\mathrm{CW}$ beam [24] is demonstrated by the data in Figure 4. The energy spread and energy centroid position, as measured by forward OTR profiling, is plotted against time for a period of two weeks, during which time there was a user requirement to keep the energy spread

small in the Hall $\mathrm{C}$ beam. The relative energy spread was held at $5 \times 10^{-5}$ during the 
whole period of running, and the viewers, in combination with a rapid digitization and analysis capability, provided the experimenters with a continuous record of the beam size. The only real problems were that for some beam parameters (low beam energy and high beam current) it is possible to trip off the machine protection system by the beam loss, and simultaneous use of the monitor and a downstream Compton beam polarimeter is sometimes not possible. All of the viewers on the Jefferson Lab IRFEL use optical transition radiation, and the viewers on the multipass beam line all have holes.

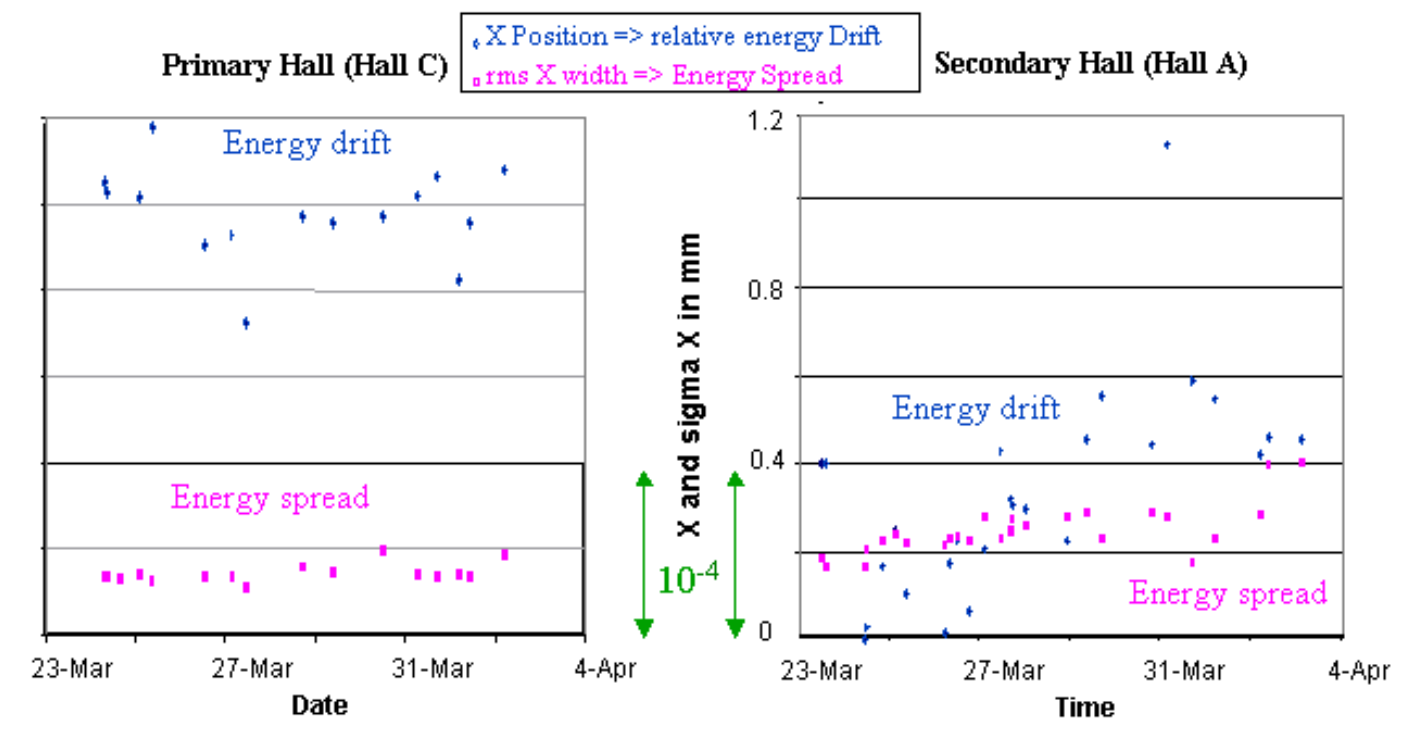

FIGURE 4. Energy Centroid and Energy Spread Measured by Optical Transition Radiation at CEBAF.

Although an experiment has been performed to check high energy diffraction transition emission by a hole on the CEBAF beam-line, no detailed experiments have yet been performed to check whether some of the theoretical details of the diffraction radiation patterns are observed in practice [25]. Diffraction radiation, as an alternative to OTR, has the nice feature that higher average beam currents may be monitored in a more non-invasive way. A technique recently developed at storage rings, involving interferometry of synchrotron radiation, is being investigated as a non-invasive continuous, and relatively fast profile monitor.

To conclude this section, it is worth mentioning that there are many wire scanners in the CEBAF accelerator. The advantages such devices have by comparison to OTR viewers are that they have somewhat better resolution than the viewers and it is thought, because electronic measurements are made, that they are more linear with the beam current. The primary disadvantages of this technique are that a single measurement takes about $45 \mathrm{sec}$, limited by the scan speed, and that one is not in a position to measure fully $\mathrm{CW}$ beam with wire scanners, limited by blinding of the machine protection systems due to loss of electrons scattered in the wire. These devices have found to be very useful for emittance measurements, establishing beam optical functions at various machine locations, and for measuring, checking, and correcting details in the beam profile. Due to slow scan speed and the extra time it 
takes to perform a full "quad-scan" emittance measurement, measurements with multiple scanners ( 4 or 5 ) at different longitudinal locations is the preferred method of doing such measurements in a machine where beam-time is a precious commodity.

\section{FUTURE WORK}

Within the past few years, work in the field of recirculated linacs has grown due to widespread interest in utilizing such accelerators in a variety of applications. Many of these applications are summarized in the review previously referenced [1] and in a recent International Committee on Future Accelerators Beam Dynamics Newsletter [26]. A substantial number of the new applications rely on applying the notion of energy recovery: the beam recirculation is arranged to have first an accelerating pass (or several accelerating passes), followed by a decelerating RF phase pass of the beam (or several passes) in order to reuse the energy resident in the beam. Such an arrangement allows substantial average beam currents to be accelerated to high energy for the application. Also, such schemes allow the total RF power cost to run the facility to be reduced far below what it would be had the linear accelerator not been energy recovered. Such statements may be quantified by defining a power multiplication factor

$$
\kappa=P_{\text {beam }}^{\text {ave }} / P_{r f}
$$

where $P_{\text {beam }}^{\text {ave }}$ is the average beam power at highest energy and $P_{r f}$ is the continuous RF power needed to accelerate the beam. It is easy to show that for a storage ring, $\kappa$ approaches the ratio of the synchrotron radiation damping time to the revolution time, a number of order or greater than a thousand for a typical ring. The most recent superconducting energy recovered linac designs have $\kappa$ from 200 to 500; the overall accelerator efficiency is getting to values approaching those in storage rings.

The other advantage of the recirculating linac arrangement has already been discussed. Just as is true for conventional linacs, the opportunity exists for manipulating the longitudinal phase space in the accelerator to produce very short electron bunches. The combination of high average beam power, produced in high frequency streams of relatively low charge electron bunches, with ultrashort bunches (of order $100 \mathrm{fsec}$ ) will probably be uniquely obtained in an energy recovered linac.

It is expected that high average current energy recovered linacs will begin to look like storage rings as regards to their electron beam diagnostics. Beam diagnosis based on detection and analysis of synchrotron light should be readily achieved with results and performance similar to present day storage rings. As the overall beam size in present ERL designs is only somewhat smaller than in storage rings, beam position monitoring and profiling will undoubtedly build on storage ring experience, and feedback and stabilization systems will undoubtedly follow storage ring developments.

New developments are anticipated in several areas: (1) beam profiling and emittance measurement at low beam energies (i.e., beam energies small enough that 
synchrotron light is not a possibility), (2) bunch length monitoring and control, especially with highly compressed bunches, and (3) measurement of electron beam halo. All of these measurements are particularly difficult if a non-invasive measurement of the high average power beam is desired.

At present it seems that the best way to diagnose an electron beam profile at low energies is to use a residual gas ionization profile monitor. Such a device can operate in a non-invasive way and preliminary calculations indicate reasonable profile integration times at the high average $\mathrm{CW}$ currents anticipated. It seems that the best way to monitor the bunch length will be through spectrum analysis of the rather copious coherent synchrotron radiation that is emitted by the high average power electron beam. An alternate idea, gaining increasing numbers of adherents recently, is to detect and spectrum analyze coherent diffraction radiation from a diffracting aperture that the high current beam can pass through without causing damage. There is interest in utilizing such diffracting holes to obtain direct measurements of beam emittance by analyzing the modulation depth of the far-field diffraction radiation pattern.

As the average circulating beam power in energy recovered linacs is huge, it will be imperative to make sure that only the smallest portion of the beam is lost in transit through the accelerator. For example, a relative beam loss less than $10^{-5}$ is necessary for a recently proposed recirculated linac light source at Cornell [27,28] to operate properly. Beam halos at this level are not routinely measured on other electron accelerators. Initial measurements will be performed on a prototype injector for this machine, one of whose purposes is to develop a strong case that high average power electron beams with small haloes can indeed be produced. Measurements will proceed by placing radiators with different hole sizes in the halo regions, and by measuring the scattered electrons by sensitive radiation detectors. It will be most important to understand which parts of the injector (where most of the halo must originate) are most important for determining halo, and finding ways to minimize the amount of halo generated.

\section{ACKNOWLEDGMENTS}

This work was accomplished through the dedicated efforts of a large range of Jefferson Lab staff. The authors would like to thank all those who helped build and perform bunch length measurements, path length measurements and adjustments, feedback systems and beam stability work, and who developed the toggling measurements into their final form. The large number of individuals involved makes it impossible to list them all here. Just the same, Karel Capek should be singled out for special thanks, as he has been involved with practically every beam diagnostics project at Jefferson Laboratory. 


\section{REFERENCES}

1. Leemann, C.W., Douglas, D. R., and Krafft, G. A., "The Continuous Electron Beam Accelerator Facility: CEBAF at the Jefferson Laboratory," in Annual Reviews of Nuclear and Particle Science, edited by C. Quigg, Palo Alto, CA: Annual Reviews, Volume 51 2001, pp. 413-450.

2. Krafft, G. A., Denard, J.-C., Dickson, R. W., Kazimi, R., Lebedev, V. A., and Tiefenback, M. G., "Measuring and Controlling Energy Spread in CEBAF" in Proceedings of the XXth International Linac Conference, edited by A. W. Chao, SLAC-R-561, Palo Alto, CA: Stanford Linear Accelerator Center, 2000, pp. 721-725.

3. Krafft, G. A., "Status of the Continuous Electron Beam Accelerator Facility" in Proceedings of the 1994 International Linac Conference, edited by K. Takata et al., Tsukuba, Japan: National Laboratory for High Energy Physics, 1994, pp. 9-13.

4. Krafft, G. A., "Correcting $M_{56}$ and $T_{566}$ to obtain very short bunches at CEBAF" in Proceedings of the Microbunches Workshop, edited by E. B. Blum, M. Dienes, and J. B. Murphy, AIP Conference Proceedings 367, New York: American Institute of Physics, 1996, pp. 46-55.

5. Krafft, G. A., Wang, D., Price, E., Feldl, E., Porterfield, D., Wood, P., and Crowe, T., "Coherent Synchrotron Radiation Detector for Non-Invasive Subpicosecond Bunch Length Monitor" in Proceedings of the 1995 Particle Accelerator Conference and International Conference on High Energy Accelerators, IEEE 95CH35843, Piscataway, NJ: Institute of Electrical and Electronic Engineers, 1995, pp. 2601-2603.

6. Wang, D. X., Krafft, G. A., Price, E., Wood, P., Porterfield, D. and Crowe, T., "A Fast Coherent Synchrotron Radiation Monitor for the Bunch Length of the Short CEBAF Bunches" in Proceedings of the Microbunches Workshop, edited by E. B. Blum, M. Dienes, and J. B. Murphy, AIP Conference Proceedings 367, New York: American Institute of Physics, 1996, pp. 502-511.

7. Wang, D. X., "Electron Beam Instrumentation Techniques Using Coherent Radiation" in Proceedings of the 1997 Particle Accelerator Conference, edited by M. Comyn et al., IEEE 97CB36167, Piscataway, NJ: Institute of Electrical and Electronics Engineers, 1998, pp. 1976-1980.

8. Wang, D. X., Krafft, G. A., Price, E., Wood, P. A. D., Porterfield, D. W., and Crowe, T. W., Appl. Phys. Letters 70, 529-531 (1997).

9. Krafft, G. A., "Diagnostics for Ultrashort Bunches" in Beam Diagnostics and Instrumentation for Particle Accelerators DIPAC 97, edited by A. Ghigo, M. Giabbai, and G. Possanza, LNF97/048(IR), Frascati: Laboratori Nazionali di Frascati, 1997, pp. 48-52.

10. Wang, D. X., and Krafft, G. A., "Measuring Longitudinal Distribution and Bunch Length of Femtosecond Bunches with RF Zero-Phasing Method" in Proceedings of the 1997 Particle Accelerator Conference, edited by M. Comyn et al., IEEE 97CB36167, Piscataway, NJ: Institute of Electrical and Electronics Engineers, 1998, pp. 2020-2022.

11. Wang, D. X., Krafft, G. A., and Sinclair, C. K., Phys. Rev. E, 57, 2283-2286 (1998).

12.Kazimi, R., Sinclair, C. K., and Krafft, G. A., "Setting and Measuring the Longitudinal Optics in CEBAF Injector" in Proceedings of the XXth International Linac Conference, edited by A. W. Chao, SLAC-R-561, Palo Alto, CA: Stanford Linear Accelerator Center, 2000, pp. 125-127.

13. Graves, W. S., private communication. See also his various contributions to Proceedings of the 2001 Particle Accelerator Conference, edited by P. Lucas and S. Webber, IEEE 01CH37268, Piscataway, NJ: Institute of Electrical and Electronics Engineers, 2001

14. Tiefenback, M. G., and Brown, K., "Beam-Based Phase Monitoring and Gradient Calibration of Jefferson Laboratory RF Systems" in Proceedings of the 1997 Particle Accelerator Conference, edited by M. Comyn et al., IEEE 97CB36167, Piscataway, NJ: Institute of Electrical and Electronics Engineers, 1998, pp. 2271-2273.

15.Krafft, G. A., Crofford, M., Douglas, D. R., Harwood, S. L., Kazimi, R., Legg, R., Oren, W., Tremblay, K., and Wang, D., "Measuring and Adjusting the Path Length at CEBAF" in Proceedings of the 1995 Particle Accelerator Conference and International Conference on High Energy Accelerators, IEEE 95CH35843, Piscataway, NJ: Institute of Electrical and Electronic Engineers, 1995, pp. 2429-2431.

16. Hardy, D., Tang, J., Legg, R., Tiefenback, M., Crofford, M., and Krafft, G. A., “Automated Path Length and $M_{56}$ Measurements at Jefferson Lab" in Proceedings of the 1997 Particle Accelerator 
Conference, edited by M. Comyn et al., IEEE 97CB36167, Piscataway, NJ: Institute of Electrical and Electronics Engineers, 1998, pp. 2265-2267.

17. Dickson, R., and Lebedev, V. A., "Fast Digital Feedback System for Energy and Beam Position Stabilization" in Proceedings of the 1999 Particle Accelerator Conference, edited by A. Luccio and W. MacKay, IEEE 99CH36366, Piscataway, NJ: Institute of Electrical and Electronics Engineers, 1999, pp. 646-648.

18.Lebedev, V. A., Musson, J., and Tiefenback, M. G., "High-Precision Beam-Based RF Phase Stabilization at Jefferson Lab" in Proceedings of the 1999 Particle Accelerator Conference, edited by A. Luccio and W. MacKay, IEEE 99CH36366, Piscataway, NJ: Institute of Electrical and Electronics Engineers, 1999, pp. 1183-1185.

19. Tiefenback, M. G., "On-line Measurement and Tuning of Multi-pass Recirculation Time in the CEBAF Linacs" in Proceedings of the 2001 Particle Accelerator Conference, edited by P. Lucas and S. Webber, IEEE 01CH37268, Piscataway, NJ: Institute of Electrical and Electronics Engineers, 2001, pp. 553-555.

20.Powers, T., Doolittle, L., Ursic, R., and Wagner, J., "Design, Commissioning, and Operational Results of Wide Dynamic Range BPM Switched Electrode Electronics" in Proceedings of the 1996 Beam Instrumentation Workshop, edited by A. Lumpkin and C. Eyberger, AIP Conference Proceedings 390, New York: American Institute of Physics, 1998, pp. 257-265.

21. Chao, Y., Crofford, M., Dobeck, N., Douglas, D., Hofler, A., Hovater, C., Krafft, G. A., Legg, R., Perry, J., Price, E., Suhring, S., Tiefenback, M., van Zeijts, J., "Commissioning and Operation Experience with the CEBAF Recirculation Arc Beam transport System" in Proceedings of the 1993 Particle Accelerator Conference, edited by S. T. Corneliussen, IEEE 93CH3279-7, Piscataway, NJ: Institute of Electrical and Electronics Engineers, 1993, pp. 587-589.

22. Lebedev, V. A., Bickley, M., Schaffner, S., van Zeijts, J., Krafft, G. A., and Watson, W. A., Nucl. Inst. and Methods A408, 373-379 (1998).

23.Piot, P., Denard, J.-C., Aderley, P., Capek, K., and Feldl, E., "High Current CW Beam Profile Monitors Using Transition Radiation at CEBAF" in Proceedings of the 1996 Beam Instrumentation Workshop, edited by A. Lumpkin and C. Eyberger, AIP Conference Proceedings 390, New York: American Institute of Physics, 1998, pp. 298-305.

24. Denard, J.-C., Piot, P., Capek, K., and Feldl, E., "High Power Beam Profile Monitor with Optical Transition Radiation" in Proceedings of the 1997 Particle Accelerator Conference, edited by M. Comyn et al., IEEE 97CB36167, Piscataway, NJ: Institute of Electrical and Electronics Engineers, 1998, pp. 2198-2200.

25. Rule, D. and Fiorito, R., "Noninterceptive Beam Diagnostics Based on Diffraction Radiation" in Proceedings of the 1996 Beam Instrumentation Workshop, Edited by A. Lumpkin and C. Eyberger, AIP conference Proceedings 390, New York: American Institute of Physics, 1998, pp. 510-517.

26. Krafft, G. A., and Zhang, Y., "Advances on Recirculated Linac Light Sources," in ICFA Beam Dynamics Newsletter No. 26, edited by K. Hirata and J. M. Jowett, International Committee for Future Accelerators, 2001, pp. 7-8, and other references throughout this newsletter.

27. Gruner, S. M., Bilderback, D., Bazarov, I., Finkelstein, K., Krafft, G., Merminga, L., Padamsee, H., Shen, Q., Sinclair, C., Tigner, M., Rev. Sci. Inst. 73, 1402-1406 (2002).

28. Bazarov, I., Belomestnykh, S., Bilderback, D., Finkelstein, K., Fontes, E., Gray, S., Gruner, S., Krafft, G., Merminga, L., Padamsee, H., Helmke, R., Shen, Q., Rogers, J., Sinclair, C., Talman, R., Tigner, M., "Study for a Proposed Phase I Energy Recovery Linac (ERL) Synchrotron Light Source at Cornell University", CHESS Technical Memo 01-003, JLAB-ACT-01-04, 2001 (unpublished) 